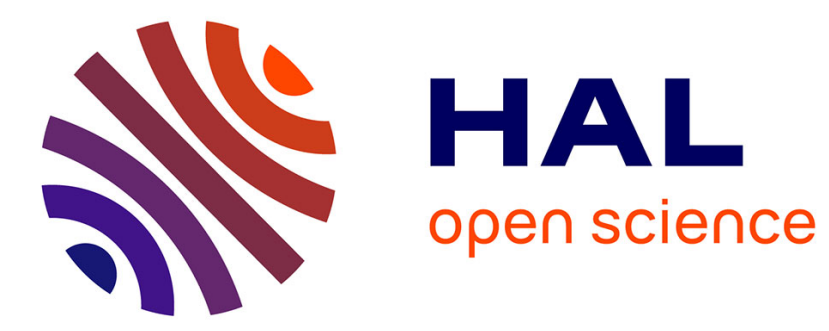

\title{
Stark ladders in strongly coupled finite superlattices
}

\author{
R. Roberts, P. Harrison, T. Stirner, W. Hagston
}

\section{To cite this version:}

R. Roberts, P. Harrison, T. Stirner, W. Hagston. Stark ladders in strongly coupled finite superlattices. Journal de Physique IV Proceedings, 1993, 03 (C5), pp.C5-203-C5-206. 10.1051/jp4:1993539 . jpa00251625

\section{HAL Id: jpa-00251625 https://hal.science/jpa-00251625}

Submitted on 1 Jan 1993

HAL is a multi-disciplinary open access archive for the deposit and dissemination of scientific research documents, whether they are published or not. The documents may come from teaching and research institutions in France or abroad, or from public or private research centers.
L'archive ouverte pluridisciplinaire HAL, est destinée au dépôt et à la diffusion de documents scientifiques de niveau recherche, publiés ou non, émanant des établissements d'enseignement et de recherche français ou étrangers, des laboratoires publics ou privés. 


\title{
Stark ladders in strongly coupled finite superlattices
}

\author{
R.G. ROBERTS, P. HARRISON, T. STIRNER and W.E. HAGSTON
}

Department of Applied Physics, University of Hull., HU6 7RX, U.K.

\begin{abstract}
It is shown that the nature and extent of wavefunction localisation of miniband states in an external electric field can be markedly different for finite superlattice structures, compared with infinite and is dependent on the strength of interaction between the initial electron (and hole) miniband states. The question of blue shifts and excitonic effects is described and possible device applications for strongly interacting superlattices structures are discussed.
\end{abstract}

\section{Introduction}

The effects of external electric fields on the band structure of semiconductor superlattices has been of considerable interest for some time[1]. A simple picture of these effects in a two band system for example, is the following. In zero electric field $(\varepsilon=0)$ the interband photoluminescence (P. L.) occurs between the bottom of the the conduction miniband and the top of the valence miniband. If the widths of these minibands are $\Delta_{c}$ and $\Delta_{v}$ repectively, it is expected that carrier localisation will occur in them at electric fields $\varepsilon_{c}=\Delta_{c} / e D$ and $\varepsilon_{v}=\Delta_{v} / e D$ where $D$ is the superlattice period. Simultaneously the transition energies would increase by an amount of the order $\left(\Delta_{c}+\Delta_{v}\right) / 2$, since the level of the isolated quantum well is, to a finite approximation, at the centre of the superlattice band. Prior to complete localisation at fields $\gtrsim \varepsilon_{c}$ (or $\dot{\varepsilon}_{v}$ ) a certain degree of delocalisation of the carrier wave functions exists, which permits electrons, say, to recombine with holes which are situated in adjacent well regions thus giving rise to Stark ladder transitions having energy separations of $\pm e \varepsilon D, \pm 2 e \varepsilon D$, etc.

Depending on the particular superlattice, this simple picture is complicated by various effects. For example does localisation of all the electron states into their respective wells occur at precisely the same value of the electric field? Is the centre of the miniband coincident with that of an isolated well and even if it is, what relevance has this to observations of the optical properties? In this connection we note that the Coulombic interaction between the electron and hole will complicate the simple picture since, in general, the value of this interaction will be different for the extended states of the superlattice (i.e. $\varepsilon=0$ ) from that when the states are localised $\left(\varepsilon \gtrsim \varepsilon_{c}\right.$ or $\left.\varepsilon_{v}\right)$. In what follows we consider two examples at the extreme ends of the miniband problem namely narrow minibands representing 'weakly' interacting quantum wells and 'broad' minibands appropriate to strongly interacting quantum wells. By this means, we are able to examine all the assumptions implicit in the simple picture just described and to point out a range of interesting phenomena that could be observed in different superlattice structures. In addition we show that strongly interacting superlattices could give rise to non-linear devices such as S.E.E.D.'s operating at much lower electric 

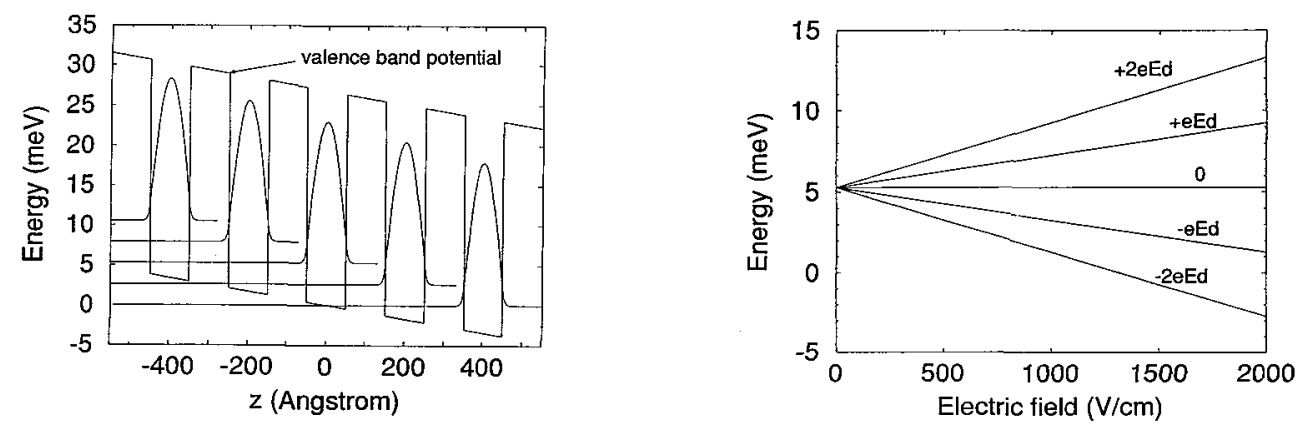

Figure 1: a)Hole wavefunctions and superlattice potential (not to scale) b) effect of field on localised hole state energies.

fields than those resulting from the 'blue shifts' occuring through the 'break-up' of minibands in 'weakly coupled superlattices'.

Since we are interested in the $\mathrm{Cd} T e / \mathrm{Cd}_{1-x} \mathrm{Mn}_{x}$ Te system we have chosen, for purposes of illustration parameters such as conduction and valence band offsets, effective masses etc. which are characteristic of these materials. However the general features which we will describe are not peculiar to such systems. The numerical calculational techniques employed have been described in earlier publications $[2,3]$.

\section{Discussion and results}

Consider first a narrow mini-band appropriate to a 5 well system and typified by the choice $x=0.5$ with well $\left(L_{W}\right)$ and barrier $\left(L_{B}\right)$ both of width $100 \AA$. Calculation shows that the corresponding field for producing localisation of, for example, the hole states is found to satisfy $\varepsilon_{v} \sim \Delta_{v} / e D$. Furthermore the hole states in the various well regions all localise at essentially the same (low) field, which implies that the wave functions are approximately symmetrical with respect to the centre of each well region. Thereafter the hole state energies vary with the field according to $\pm e E d, \pm 2 e E d$ etc. These features are shown in Figures $1 \mathrm{a}$ and $1 \mathrm{~b}$.

As opposed to this the behaviour of a strongly coupled superlattice is much more complex. In order to illustrate this we consider an eleven well system for which $x=0.075, L_{W}=20 \AA$, and $L_{B}=20 \AA$. Such a system can in zero field support 10 bound states in the valence band and 5 bound states in the conduction band. The corresponding miniband widths are wide with the individual one-electron
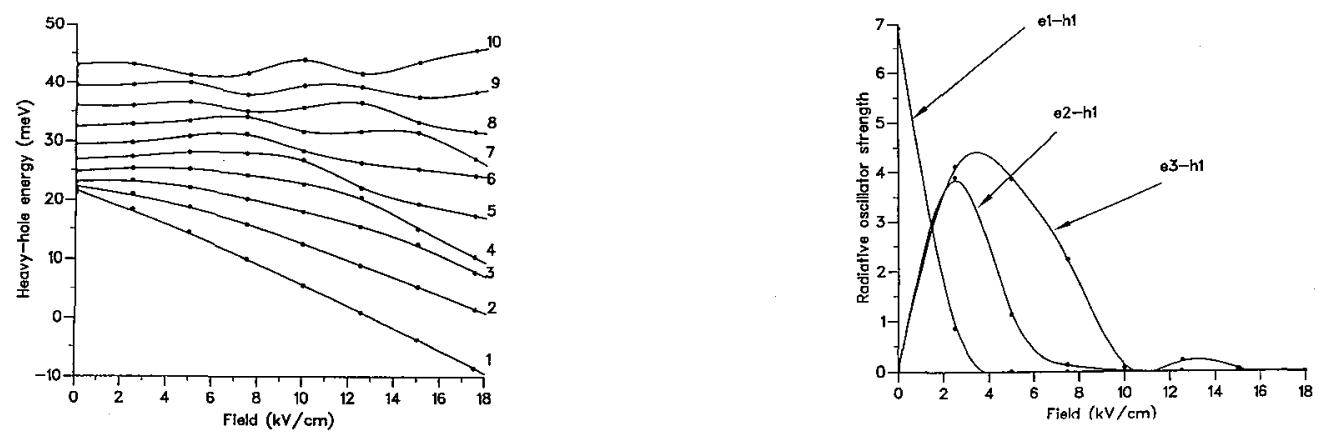

Figure 2: Effect of electric field on a) hole energies and b) oscillator strengths of selected excitonic transitions. 

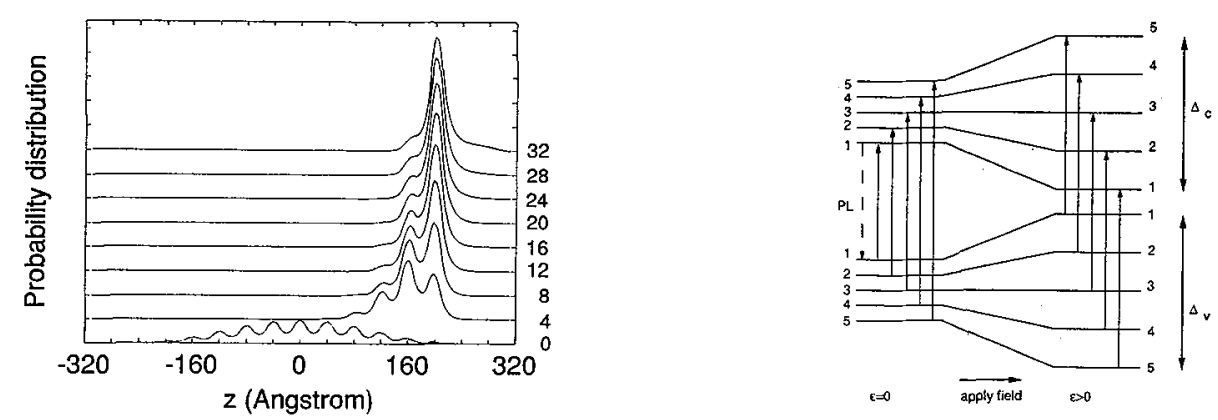

Figure 3: a)Effect of electric field (right hand axis, in units of $\mathrm{kVcm}^{-1}$ ) on the hole wavefunction and $b$ ) schematic representation of origin of blue shift

energies for the hole being as shown in Figure 2a. (The miniband centre varies with the field and does not coincide with the isolated well energy.) Typical values of the oscillator strengths of various transitions corresponding to electron-hole excitonic transitions between the various miniband states are shown as a function of field in Figure 2b. In order to appreciate the significance of this curve we show in Figure 3a the probability distribution of the lowest hole state for various values of the electric field. At zero field the hole is distributed across the whole superlattice structure however we note that, as opposed to the equal probability distribution of an infinitely long superlattice, the hole has a greater probability of being found in the central well region of a finite superlattice. With increasing field the hole wave function moves to the right of the structure and at a field $\varepsilon \sim \varepsilon_{v}$ the hole is still spread over two well regions (i.e. it is not fully localized). This delocalization persists to much higher fields, and is even more extensive for the higher energy hole states. Similar effects are found for the electron miniband states although now the lowest energy electron state moves over to the left of the structure. It is this pulling apart of the various electron and hole states that is responsible for the shape of the oscillator strength curves shown in Figure $2 \mathrm{~b}$.

This same feature also permits a clearer understanding of the nature of the blue shifts that occur on application of an electric field. In order to illustrate this we consider a 5 well structure and invoke the hypothetical situation of an equally spaced conduction and valence miniband structure, as in Figure 3b. Labelling the lowest energy electron (hole) states in the sequence 1 to 5 we see that the lowest electron (1) and the highest hole (5) state will localize in the first well followed by the next lowest electron (2) and next highest hole state (4) and so on. The effect of all this is that for example the photoluminescence (PL) would originally in zero field correspond to the transition $1 \rightarrow$ 1 whereas, following localization, the lower energy direct intra-well transitions are blue shifted, the higher ones red shifted and correspond to exactly the same energy (i.e. $1 \rightarrow 5,2 \rightarrow 4,3 \rightarrow 3,4 \rightarrow$ $2,5 \rightarrow 1$ ). This blue shift would be exactly one half of the sum of the (identical) miniband widths.

Two other important features of the more detailed calculations can also be illustrated by simple considerations of this nature. In zero field a given quantum well is symmetrical with respect to (w.r.t.) its centre point. This means that the wave functions satisfy $\psi(z)= \pm \psi(-z)$ and $|\psi(z)|^{2}=|\psi(-z)|^{2}$, thus giving a symmetrical probability distribution. If the inversion symmetry of the quantum well is destroyed, as for example in an electric field, then $\psi(z) \neq \pm \psi(-z)$ and $|\psi(z)|^{2} \neq|\psi(-z)|^{2}$ - i.e. the probability distribution becomes skewed w.r.t. the centre of the well. Clearly if the electron distribution is skewed to the left of centre then the hole distribution will be skewed to the right of centre. Such a polarization will cause the intra-well transition to move in a field, but more important is the effect it has on inter-well transitions. Consider for example an electron in well 0 recombining with holes in nearest neighbour well positions \pm 1 . Given that the electron is to the left of centre, whilst the hole is to the right, it is clear that the electron will be closer to the hole on its left (well 


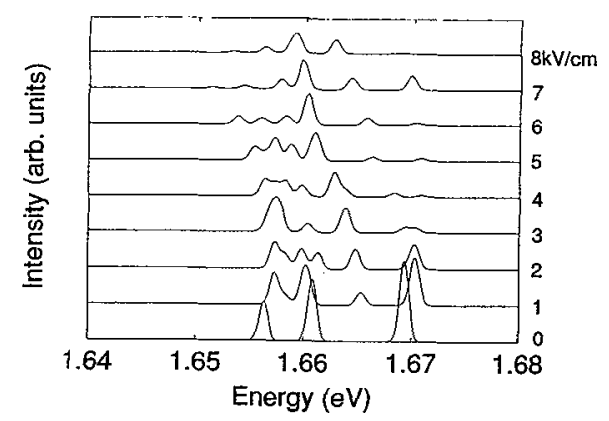

Figure 4: Effect of field on exication spectra

-1 say) than it will to the hole on its right (well +1 say). These differences in wave function overlap can result in marked differences in the oscillator strengths and exciton binding energies for the $0 \rightarrow$ -1 exciton compared with the $0 \rightarrow+1$ exciton. Such differences were evident to varying degrees in our calculations for various superlattice structures, and can clearly complicate the simple picture described previously.

One final point related to several of the features we have described concerns a novel device application for strongly interacting superlattices. Basically non-linear optical devices such as S.E.E.D.'s work on the principle that absorption at a given wavelength can be reduced via an external electric field. This can be achieved by e.g. blue shifting the absorption line. However the device would work just as effectively if the energy position of the line remained the same but its oscillator strength was reduced. We will show that the strongly interacting finite quantum well system appropriate to Figures $2 \mathrm{a}$ and $2 \mathrm{~b}$ combines both these aspects. On the assumption that the full width at half maximum of an absorption line corresponds to $\approx 1 \mathrm{meV}$ we have constructed in Figure 4 the absorption spectra as a function of field. The main point we draw attention to here concerns the lowest energy absorption line. With increasing field this line moves initially to higher energy and decreases rapidly in intensity. The latter results from the electron and hole being moved in opposite directions, whilst the initial blue shift results from the exciton binding energy decreasing more rapidly as a result of this movement than the decrease in the one-particle energies. These effects occur at field values far removed from those required to produce localization. Since the line intensity decreases rapidly at such low fields this could have clear device possibilities for S.E.E.D.'s.

\section{Conclusion}

We have shown that a variety of interesting physical effects resulting from wave function localization could occur in different superlattice structures. Such effects not only complicate the simple interpretation of Stark ladder formation but have the potential for exploitation in device structures.

\section{References}

[1] M. K. Saker, D. M. Whittaker, M. S. Skolnick, M. T. Emeny, and C. R. Whitehouse, Superlattices and Microstructures 10295 (1991) and references therein.

[2] C. P. Hilton, J. P. Goodwin, P. Harrison and W. E. Hagston, J. Phys. A25 2395 (1992)

[3] C. P. Hilton, W. E. Hagston and J. E. Nicholls, J. Phys. A25 5365 (1992) 